\title{
O SUS nos seus 20 anos: reflexões num contexto de mudanças
}

\section{Twenty years of SUS: reflections in a context of changes}

\section{Georgia Costa de Araújo Souza}

Cirurgiã-Dentista. Mestre em Odontologia.

Endereço: Rua Joaquim Araújo Filho, 1440, Lagoa Nova, CEP 59063120, Natal, RN, Brasil.

E-mail: georgia_odonto®yahoo.com.br

Iris do Céu Clara Costa

Cirurgiã-Dentista. Professora Doutora do Programa de Pós-Graduação em Odontologia da Universidade Federal do Rio Grande do Norte - UFRN.

Endereço: Av. Senador Salgado Filho, 1787, Lagoa Nova, CEP 59056600, Natal, RN, Brasil.

E-mail: iris_odontoufrnळyahoo.com.br

\section{Resumo}

A partir de uma breve retrospectiva sobre o direito à saúde conquistado pelo cidadão brasileiro, este artigo tem como objetivo discorrer sobre o Sistema Único de Saúde (SUS) ao longo da sua trajetória de 20 anos, destacando em forma de análise crítica os avanços conquistados na saúde e os desafios a serem superados. São discutidas as temáticas do direito à saúde como responsabilidade do Estado a partir da Conferência de Alma-Ata, da Constituição Federal e das Leis Orgânicas da Saúde; as diretrizes do SUS envolvendo as competências da União, do Estado e do Município. Discute ainda a importância da Estratégia Saúde da Família na consolidação da Atenção Básica no Brasil, as dificuldades do processo de regionalização e o papel da gestão e da participação social como bases para a construção do SUS que queremos.

Palavras-chave: Sistema Único de Saúde; Saúde Pública; Política Nacional de Saúde. 


\section{Abstract}

From a brief review of the right to healthcare won by the Brazilian citizens, this article aims to discuss Sistema Unico de Saúde (SUS - National Health System) throughout its trajectory of 20 years, providing a critical review of the progress achieved in healthcare and the challenges to be overcome. It discusses the issues of: the right to healthcare as the State's responsibility based on the Alma-Ata Conference, the Federal Constitution and the Organic Laws on Health; SUS guidelines involving the competences of the Union, States and Municipalities. It also approaches the importance of the Family Health Strategy for the consolidation of Primary Care in Brazil, the difficulties in the process of regional health planning, and the role of management and social participation as the bases for the construction of the SUS we want.

Keywords: National Health System; Public Health; National Health Policy.

\section{Um Resgate Histórico do Direito à Saúde como Responsabilidade do Estado}

0 atual sistema de saúde brasileiro vive um momento de intensos avanços, mas ainda de muitos desafios a serem superados. Podemos descrever como avanços o que se refere à oferta de diversos programas, projetos e políticas que têm apresentado resultados inegáveis e exitosos para a população brasileira, que incluem a evolução das equipes do Programa Saúde da Família, do Programa Nacional de Imunizações, do Sistema Nacional de Transplantes, sendo o segundo país do mundo em número de transplantes, do Programa de Controle de HIV/AIDS, reconhecido internacionalmente pelo seu progresso no atendimento às Doenças Sexualmente Transmissíveis/AIDS, entre outros (Brasil, 20o6b). Como desafios, podemos enumerar aqueles referentes aos problemas de implementação, implantação, financiamento e gestão do Sistema Único de Saúde (SUS), fato que, para ser compreendido, merece uma análise mais detalhada, fazendo-se necessário um resgate do processo de construção do sistema de saúde no Brasil a partir da construção do SUS.

A criação do Sistema Único de Saúde foi o maior movimento de inclusão social já visto na História do Brasil e representou, em termos constitucionais, uma afirmação política de compromisso do Estado brasileiro para com os direitos dos seus cidadãos (Brasil, 2007c). A partir da Conferência Internacional sobre Cuidados Primários de Saúde, realizada em Alma-Ata (Cazaquistão, antiga URSS), no ano de 1978, ficou estabelecido num plano mundial, através do documento final deste evento, a Declaração de Alma-Ata, a participação efetiva dos Estados na saúde do seu povo através da promoção de políticas de saúde que visassem o bem-estar físico, mental e social como direitos fundamentais dos seus habitantes, enfatizando-se principalmente os cuidados primários. Evidenciou-se também que a saúde é a mais importante meta social mundial e que, para a sua realização, faz-se necessária a integração com os diversos setores sociais e econômicos (Ventura, 2003). Paralelamente a esse acontecimento histórico da saúde mundial, o Brasil passava por um momento de clamor coletivo por mudanças políti- 
cas voltadas para a redemocratização do país, que se intensificaram na década de 1980 por meio de manifestações populares pela eleição direta de um presidente civil, e, no campo da saúde, voltado para uma atenção abrangente, democrática e igualitária, tendo como principais atores sociais os intelectuais, as lideranças políticas, os profissionais da saúde, os movimentos estudantis universitários, os movimentos sindicais, entre outros, o que culminou com o esgotamento do modelo médico assistencial privatista vigente (Medeiros Júnior e Roncalli, 2004).

Nos anos seguintes, a população brasileira continuou imersa em diversos conflitos sociais, com profundas desigualdades, alto índice de desemprego, grande contingente de miseráveis, enfrentamento de filas infindáveis na busca da assistência à saúde, culminando com uma grande insatisfação popular.

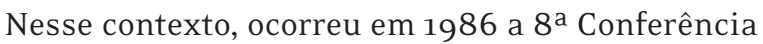
Nacional de Saúde, que contou com uma intensa participação popular de mais de 4000 pessoas, dentre as quais 1000 eram delegados com direito a voz e a voto (Brasil, 1986).

Essa Conferência se tornou um dos grandes marcos da saúde no Brasil, podendo ser considerada como o marco inicial da Reforma Sanitária brasileira. Trouxe à tona temas como: a necessidade de ampliação do conceito de saúde e de um novo Sistema Nacional de Saúde, a separação de "Saúde" da "Previdência" e a orientação da política de financiamento para o setor saúde (Brasil, 1986). Seu relatório final ficou consolidado como um instrumento que veio influenciar as responsabilidades do Estado em assegurar o direito à saúde para toda a população, garantindo condições de acesso e qualidade dos serviços, servindo de subsídio para a elaboração da nova Constituição de Saúde do Brasil (Brasil, 1988), além de estabelecer como principal objetivo a ser alcançado um sistema de saúde com atribuições e competências para os níveis Federal, Estadual e Municipal, o que culminou na construção do Sistema Unificado e Descentralizado de Saúde (SUDS) como uma necessidade imediata e de transformação progressiva para o Sistema Único de Saúde (SUS).

\section{A Constituição Federal e as Leis Orgânicas da Saúde: um entendimento dessa relação}

Conforme descrito, a Constituição Federal de 1988 consagrou a saúde como "direito de todos e dever do Estado, garantida mediante políticas sociais e econômicas que visam à redução do risco de doença e de outros agravos e possibilitando o acesso universal e igualitário às ações e serviços para promoção, proteção e recuperação" (Brasil, 1988). Essa definição conceitual adquire sua versão prática com a instituição formal do SUS, quando no Capítulo II, artigo 198 da mesma Constituição, é relatado que as ações e os serviços públicos de saúde integram uma rede regionalizada, hierarquizada e constituem um sistema único, organizado de acordo com as diretrizes: descentralização, atendimento integral e participação da comunidade. Assim, estava criado o Sistema Único de Saúde, resultado de uma política social e universalista, que tem a Constituição Federal e as Leis $n^{0} 8.080$ e $n^{0} 8.142$, ambas de 1990 , como sua base jurídica, constitucional e infraconstitucional (Brasil, 2007a).

A Lei $n^{\circ} 8.080$, de 19 de setembro de 1990 - Lei Orgânica da Saúde -, dispõe acerca das condições para a promoção, proteção e recuperação da saúde, organização e funcionamento dos serviços correspondentes, mostrando de forma clara os objetivos do SUS, suas competências e atribuições, assim como as funções da União, dos Estados e dos Municípios (Brasil, 1990a). A Lei no 8.142, de 28 de dezembro de 1990, dispõe sobre a participação da comunidade na gestão do SUS e sobre as transferências intergovernamentais de recursos financeiros na área da saúde (Brasil, 199ob). Tais leis consolidam o papel do município como o principal executor das ações de saúde, caracterizando ampliação do processo de descentralização que já havia sido exposto desde a $8^{\mathrm{a}}$ Conferência de Saúde e que significava um avanço e contraposição ao modelo ditatorial vigente naquele momento. 


\section{O Sistema Único de Saúde e suas Diretrizes: definindo competências da União, dos Estados e dos Municipios}

No nível federal, a centralização da saúde tratava com distanciamento as questões mais particulares de cada localidade, existindo um único eixo norteador para as ações de saúde em todo o país indistintamente. No entanto, a descentralização, entendida como forma de transferência de recursos e delegação de funções entre os níveis de governo (Arretche, 2002), entra em vigor com o movimento de reforma e estabelece que os serviços de saúde podem ser melhor coordenados e atender de modo mais preciso aos anseios e necessidades dos usuários através da municipalização.

Uma das diretrizes do SUS, a descentralização tem sua aplicação através do financiamento a cargo das três esferas de governo e a execução de serviços por conta dos municípios. Esse processo de descentralização no Brasil envolve a transferência de serviços, responsabilidades, poder e recursos da esfera federal para a municipal. Ao longo da década de 1990, foi verificada uma grande aceitação dos municípios pela municipalização, a qual pode ser explicada pelo interesse em aumentar a renda orçamentária de suas receitas, o que é perceptível quando, no ano de 2000, 99\% dos municípios estavam habilitados no Sistema Único de Saúde (Arretche, 2002).

Alguns municípios brasileiros dispõem de capital para o setor saúde advindo quase que exclusivamente através desse repasse, não apresentando condições de prover sua área territorial com todas as ações e serviços necessários para a atenção integral de sua população. Por isso, a regionalização torna-se necessária para que as pessoas possam buscar soluções aos seus problemas de saúde nos municípios-polo, mesmo que distantes do seu local de moradia.

Através da descentralização, busca-se envolver todas as esferas do governo para que, juntas, funcionem no sentido de promover melhorias na situação de vida e de saúde da população. Entretanto, um problema ocorre quando o repasse financeiro para estados e municípios não é administrado com responsabilidade e grande parte das finanças não é aplicada no setor saúde, recursos estes que poderiam ser investidos em contratação e capacitação de profissionais, materiais e tecnologias de saúde. Por isso, ao se propor a descentralização, seria conveniente discorrer sobre a capacitação da gestão, o que inclui a correta aplicação de recursos financeiros no setor saúde, para que avanços reais não sejam mera utopia.

A esse respeito, as Normas Operacionais Básicas (NOB) 91, 93 e 96 e as Normas Operacionais de Assistência à Saúde (NOAS) tiveram um importante papel no processo de descentralização, na medida em que definiram competências, responsabilidades e condições necessárias para que estados e municípios pudessem assumir as condições de gestão no SUS (Viana e col., 2002).

Conforme afirmam Viana e colaboradores (2002), as análises sobre o processo recente de descentralização apontam, na área social, e, em específico, na saúde, a tendência das variáveis microinstitucionais (poderes locais) terem respondido de forma mais direta pelo (in)sucesso de determinadas políticas, evidenciando um grau de autonomia da gestão local.

Outra diretriz do SUS refere-se ao atendimento integral, com prioridade para as atividades preventivas. A integralidade corresponde a um dos grandes desafios do SUS e diz respeito a tratar cada pessoa como um ser indivisível e integrante de uma comunidade; às ações de promoção, proteção e recuperação da saúde que formam um todo indivisível que não pode ser compartimentado; e às unidades prestadoras de serviço, com seus diversos níveis de atenção à saúde, Básica, Secundária e Terciária, que formam uma unidade configurando um sistema capaz de prestar assistência integral (Brasil, 1990c).

A integração das ações remete à continuidade do atendimento e ao cuidado dos usuários em seus diversos níveis, o que deve ser regulado palas unidades prestadoras de serviço. No entanto, essa integração por diversas vezes e maneiras não ocorre, seja por descompromisso daqueles que trabalham, por dificuldades impostas pelas unidades e, ainda, por uma série de razões que deixam a população sem a devida atenção e continuidade de tratamento e sem conhecimento dos seus direitos.

A garantia dos direitos constitui-se na democracia participativa em que os cidadãos influenciam 
na definição e execução das políticas de saúde (Vasconcelos e Pasche, 2006). Essa participação da comunidade na gestão do SUS, por meio do controle social, amparada pela Lei 8.142 de 1990 (Brasil, 1990b), diz respeito à representação dos usuários no processo de participação do fazer e pensar saúde, seja nas Unidades de Saúde, nos Conselhos ou nas Conferências de Saúde que ocorrem oportunamente nas três esferas de governo.

O Sistema Único de Saúde, como responsável por ações de promoção, prevenção e recuperação de saúde, apresenta propostas legislativas completas para um sistema de saúde nacional. Porém, num país com dimensões territoriais continentais como o Brasil, que enfrenta uma série de desafios sociais, econômicos, políticos, com inúmeras desigualdades, sua efetivação torna-se de difícil realização. São diversos os problemas a enfrentar, a começar pela situação de vida dos cidadãos. De acordo com o conceito ampliado de saúde da Organização Mundial de Saúde, "saúde é um estado de completo bem-estar físico, mental e social e não somente a ausência de doença”, envolvendo questões como emprego, lazer, educação, moradia, saneamento, entre outras (WHO, 1946). Muito embora esse conceito seja tomado como mera utopia, pois algum grau de doença é compatível com o estado de saúde, é imprescindível considerar a participação dos determinantes socioeconômicos no processo saúde-doença. Desse modo, é mister uma integração entre o setor saúde com diversos outros para se alcançar o estado de saúde de fato e de direito.

\section{Limitações e Desafios a Enfrentar na Consolidação do SUS}

Pode-se dizer que o SUS enfrenta uma problematização dicotômica entre o que está escrito e o que é realizado. A descrença do povo brasileiro num sistema de saúde para todos leva milhões de pessoas a procurar por serviços, planos ou seguradoras de saúde privados, pagando, por conseguinte, abusivos valores, especialmente para a população de faixa etária mais avançada que, em função do aumento da expectativa de vida e dos agravos da terceira idade, é a que mais necessita. Esse fato sustenta-se na perspectiva de que um dos temas com maiores demandas recebidas pela Agência Nacional de Saúde Suplementar (ANS) consiste no aumento da mensalidade de operadoras e planos de saúde (Brasil, 20o7b). Porém, o sistema de saúde suplementar é importante num país com desigualdades sociais acentuadas, como o Brasil, onde existe uma grande concentração de renda, tornando-se necessária a utilização do sistema privado por aqueles que possam, o qual tem estabelecido maiores parcerias com o SUS, em caráter complementar de ações, devido à insuficiência na disponibilidade e oferta de determinados serviços públicos. Essa participação do setor privado no SUS é mais pronunciada na atenção hospitalar e na oferta de serviços especializados de alto custo e densidade tecnológica, que o sistema público não pôde alcançar devido à insuficiência de investimentos (Vasconcelos e Pasche, 2006).

A complexidade do SUS, as dificuldades locorregionais, a fragmentação das políticas e programas de saúde, a qualificação da gestão e do controle social, a organização de uma rede regionalizada e hierarquizada de ações e serviços de saúde têm se constituído em desafios permanentes na consolidação do Sistema Único de Saúde.

A dificuldade dos gestores para promover a integração entre estados, municípios e as redes assistenciais estatais com os serviços de abrangência nacional tem levado a problemas no acesso aos serviços e ao comprometimento da universalidade e integralidade (Vasconcelos, 2005). Assim, diversos usuários não usufruem do direito universal à saúde. A existência de gestores mais preocupados com campanhas políticas eleitorais do que com a saúde dos seus cidadãos leva a falhas graves no sistema e o mais prejudicado com isso é o próprio povo. Nesse sentido, a melhoria na gestão reflete-se, consequentemente, num melhor aporte financeiro do setor, com um equânime repasse de recursos, melhor utilização e aplicação desse investimento, maior remuneração salarial para os profissionais da saúde, com o possível estabelecimento de vínculos, hoje ainda bastante precarizados, gerando desmotivação e desestímulo entre os servidores.

Não obstante, a precarização do trabalho desagrada os profissionais da rede que, por diversas razões, não recebem salários justos, não têm vínculos empregatícios nem direitos trabalhistas. Essa 
situação profissional pode culminar na realização de um trabalho sem compromisso com a comunidade, com a ausência de vínculos profissionais-usuários tão preconizados pela Estratégia Saúde da Família (ESF), gerando uma relação fantasiosa entre empregador/empregado, do tipo "você faz de conta que me paga e eu faço de conta que trabalho".

A Estratégia Saúde da Família como reestruturante da Atenção Básica responde por este nível de atenção no SUS e pelo encaminhamento dos atendimentos que exijam média e alta complexidade - sistema de referência - para os níveis de atenção Secundária e Terciária. Em diversos municípios brasileiros, a ESF não funciona de forma integral e equitativa, o que acontece por diversos motivos, dentre os quais podemos destacar: a contratação de profissionais sem perfil para esse nível de atenção; a precarização do trabalho ou, ainda, a ineficiente administração de gestores que induzem os profissionais a realizarem ações meramente curativas, visando atender o maior número de pessoas para que isso seja mostrado à sociedade como uma atuação da política local, tendo em vista as campanhas eleitorais. Tais ações ainda são muito valorizadas pela população em geral que sempre "recebeu" a saúde na sua maneira assistencial-curativista, e que, portanto, ainda não percebe o verdadeiro sentido da saúde como um bem-estar biopsicossocial garantido a partir de ações de prevenção e promoção de saúde.

Nessa perspectiva, a formação dos profissionais da saúde compõe o conjunto das atividades que precisam ser continuamente revistas, minimizando situações em que o profissional não tenha perfil para o trabalho coletivo, integrado, interdisciplinar, entre outros, necessário para o SUS que queremos construir. Segundo Amâncio Filho (2004), é indispensável construir um processo educacional que articule a formação profissional com as necessidades e demandas da sociedade, como estratégia para o desenvolvimento econômico, social e cultural. Por conseguinte, essa articulação deve ocorrer desde o momento da graduação ou da formação técnica, até momentos posteriores à inclusão dos profissionais no serviço, através de estratégias de educação permanentes.

Atualmente, algumas Universidades vivenciam o momento de mudanças curriculares nos Cursos da Saúde, mudanças essas na maioria dos casos estreitamente relacionadas com o SUS. Aliado a isso, o Ministério da Saúde está investindo em caráter nacional na educação permanente, até mesmo através da estratégia da Educação à Distância, na perspectiva de minimizar o fosso existente entre a academia e os serviços, vencer limitações na busca de conhecimentos complementares que auxiliem o desempenho de funções, especialmente quando se trata de gestão (Costa, 2007).

Além da gestão, é necessária a qualificação do controle social, envolvendo as múltiplas ações que as forças sociais desenvolvem para influenciar a formulação, execução, fiscalização e avaliação das políticas públicas e dos serviços no campo da saúde (Gamarra Júnior, 2006). Nesse sentido, Gamarra Júnior (2006) expõe como desafios a serem superados sobre o controle social os problemas de formação dos conselheiros, a dificuldade dos cidadãos para conseguir acesso às informações do sistema, a falta de cumprimento das deliberações dos conselhos por parte dos gestores, o desconhecimento por parte da grande massa da população dos seus direitos como controladores do SUS, entre outros. Dessa forma, fundamental se faz a qualificação dos conselhos de saúde, ampliando-os em locais onde já existam e implantando-os em locais onde ainda não ocorrem.

Essas e outras limitações que o SUS enfrenta merecem e precisam ser do conhecimento de todos para que se busquem alternativas e sólidas resoluções, como sugere Campos (2007) através de um movimento em defesa de políticas sociais e de distribuição de renda, da apresentação do SUS para a sociedade como uma reforma social significativa com impactos sobre o bem-estar e a proteção social e do seu desempenho concreto, capaz de melhorar as condições sanitárias e de saúde das pessoas. Esse se configura no maior desafio com relação ao SUS.

As mudanças pelas quais o SUS está sendo conduzido representam potenciais desafios aos gestores em todas as esferas de governo. 0 estabelecimento do Pacto pela Saúde, a Regionalização solidária e cooperativa, a Política Nacional de Humanização, entre outras, constituem-se ações que buscam a superação dos principais entraves do sistema.

No ano de 2006, foi estabelecido o Pacto pela Saúde, que introduz um sentido de gestão pública 
por resultados e de responsabilização sanitária, aprofundando a descentralização para estados e municípios, através de um acordo interfederativo articulado em três dimensões: o Pacto em Defesa do SUS, que representa um movimento social em defesa do SUS; o Pacto pela Vida, que reforça o movimento da gestão pública por resultados, com prioridades para a saúde do idoso, a redução da mortalidade infantil e materna, o controle das doenças emergentes ou endemias, a redução das vítimas por câncer de colo de útero e de mama, a promoção da saúde e o fortalecimento da Atenção Primária; e o Pacto de Gestão que estabelece responsabilidades mais claras para cada ente federado, com regionalização integrada das ações e serviços de saúde (Brasil, 2007a, Brasil, 2006b). O Pacto pela Saúde representa um compromisso público com perspectiva de superar as dificuldades enfrentadas pelo SUS dando ênfase às necessidades de saúde da população. Constituise num conjunto de reformas institucionais do SUS pactuado entre as três esferas de gestão (União, Estados e Municípios) com o objetivo de promover inovações nos processos e instrumentos de gestão, visando alcançar maior eficiência e qualidade das respostas do Sistema Único de Saúde.

As propostas de descentralização e regionalização vêm sendo experimentadas no Brasil desde a Constituição Federal de 1988, com atribuição da autonomia dos três entes federados. Entretanto, os entraves na aplicabilidade dessas diretrizes nortearam para a necessidade do estabelecimento de mecanismos e instrumentos de coordenação e cooperação que favoreçam as ações intergovernamentais no setor saúde. A gestão descentralizada da saúde ainda enfrenta o desafio de encontrar mecanismos que superem a fragmentação das estruturas municipais organizadas de modo muitas vezes estanque, induzindo iniquidades na qualidade e no acesso aos serviços, fato este que compromete a legitimidade do sistema (Brasil, 20o6a).

A operacionalização dessas diretrizes caminha, na atualidade, para a concretização do Plano Diretor de Regionalização (PDR), o qual deve traçar o desenho final do processo de pactuação entre os gestores, identificando e reconhecendo as Regiões de Saúde, desencadeando ações de construção e reconstrução, visando potencializar as ações de saúde em âmbito regional (Brasil, 2006a). Consiste numa ação que vem sendo trabalhada pelas Secretarias Estaduais de Saúde, as quais enfrentam alguns entraves na implementação em virtude da magnitude envolvida no processo de regionalização, como a articulação intermunicipal, as pactuações entre municípios e Estados, o repasse financeiro, as formas de acesso aos serviços pelos usuários e os processos de gestão locorregionais.

Com relação à Política Nacional de Humanização da Atenção e Gestão no Sistema Único de Saúde, esta vem sendo entendida como um caminho favorável à superação de desafios na saúde, através da ampliação do acesso com qualidade aos serviços e bens de saúde, da corresponsabilização entre trabalhadores, gestores e usuários nos processos de gestão e atenção, do apoio à construção de redes cooperativas, solidárias e comprometidas com a produção de saúde e com a produção de sujeitos (Brasil, 2008).

Entende-se, portanto, o SUS como uma política viva que se encontra em pleno curso de mudança e construção voltadas para a efetivação da saúde da população brasileira por meio dos princípios da universalidade, integralidade e equidade, e para o enfrentamento dos desafios vivenciados ao longo de sua trajetória de 20 anos.

\section{À Guisa de Conclusões}

O Sistema Único de Saúde representa avanços e conquistas na saúde de todos os brasileiros e, comemorando seus 20 anos de existência, conseguiu enfrentar muitas barreiras, o que resultou, sobretudo, no direito da população brasileira de contar com um serviço de atenção à saúde. Sendo um sistema de saúde que está em construção, passa por inúmeras mudanças para efetivação dos seus ideários, tendo como pressupostos a promoção, proteção e recuperação da saúde.

O SUS está consolidado no nosso país, mas enfrenta desafios constantemente, dentre os quais, a qualificação da gestão e do controle social, o fortalecimento e a qualificação da Atenção Básica como estratégia organizadora das redes de cuidado em saúde, as dificuldades no acesso às ações e serviços de saúde, a fragmentação das políticas e programas de saúde, a organização de uma rede 
regionalizada e hierarquizada de ações e serviços de saúde, o reconhecimento da autonomia dos entes federados, entre outros. Esses dilemas conjunturais desafiam os gestores e precisam ser assumidos como responsabilidades para a concretização do SUS que queremos.

Ao longo desse tempo, vimos que não é fácil tornar práticos os seus princípios de universalidade, integralidade e equidade. Vislumbra-se, assim, para o enfrentamento dos desafios, a articulação entre os atores envolvidos, gestores, servidores e usuários, com o desejo de fazer o melhor para a população brasileira, cabendo a todos os comprometidos trabalharem em prol do êxito do SUS, a fim de que as políticas públicas sejam bem aplicadas e possam constituir meios que promovam a qualidade de vida das pessoas.

\section{Referências}

AMÂNCIO FILHO, A. Dilemas e desafios da formação profissional em saúde. Interface, Botucatu, v. 8, n. 15, p. 375-380, 2004.

ARRETCHE, M. Relações federativas nas políticas sociais. Educação \& Sociedade, Campinas, v. 23, n. 8o, p. 25-48, 2002.

BRASIL. Ministério da Saúde. Relatório final da $8^{a}$ Conferência Nacional de Saúde. Brasília, DF, 1986.

BRASIL. Constituição da República Federativa do Brasil de 1988. Diário Oficial da União, Brasília, DF, 5 out. 1988. Seção II, p. 33-34.

BRASIL. Lei n. 8.o8o, de 19 de setembro de 1990. Dispõe sobre as condições para a promoção, proteção e recuperação da Saúde, a organização e o funcionamento dos serviços correspondentes e dá outras providências. Diário Oficial da União, Brasília, DF, 20 set. 1990a. Seção 1, p. 1805518059.

BRASIL. Lei n. 8.142, de 28 de setembro de 1990. Dispõe sobre a participação da comunidade na gestão do Sistema Único de Saúde (SUS) e sobre as transferências intergovernamentais de recursos financeiros na área da Saúde e dá outras providências. Diário Oficial da União, Brasília, DF, 31 dez. 199ob. Seção 1, p. 25694-25695.
BRASIL. Ministério da Saúde. Secretaria Nacional de Assistência à Saúde. $A B C$ do SUS. Brasília, DF, 1990c.

BRASIL. Ministério da Saúde. Departamento de Apoio à Descentralização. Regionalização solidária e cooperativa. Brasília, DF, 2006a. (Série Pactos pela Saúde, 6).

BRASIL. Portaria GM/MS 399 de 22 de fevereiro de 2006. Divulga o Pacto pela Saúde 2006 consolidação do SUS e aprova as diretrizes operacionais do referido pacto. Diário Oficial da União, Brasília, DF, 23 fev. 20o6b. Seção 1, p. 43-51.

BRASIL. Conselho Nacional de Secretários de Saúde. Legislação estruturante do SUS. Brasília, DF, 2007a. (Coleção Progestores - Para entender a Gestão do SUS, 12).

BRASIL. Conselho Nacional de Secretários de Saúde. Saúde suplementar. Brasília, DF, 2007b. (Coleção Progestores - Para entender a Gestão do SUS, 11).

BRASIL. Conselho Nacional de Secretários de Saúde. Sistema Único de Saúde. Brasília, DF, 2007c. (Coleção Progestores - Para entender a Gestão do SUS, 1).

BRASIL. Ministério da Saúde. Secretaria de Atenção à Saúde. Núcleo Técnico da Política Nacional de Humanização. HumanizaSUS: documento base para gestores e trabalhadores do SUS. 4. ed. Brasília, DF, 20o8. (Série B: Textos Básicos de Saúde).

CAMPOS, G. W. S. Reforma política e sanitária: a sustentabilidade do SUS em questão? Ciência e Saúde Coletiva, Rio de Janeiro, v. 12, n. 2, p. 301306, 2007.

COSTA, I. C. C. Os sete saberes necessários à educação do futuro e o planejamento das ações de saúde: algumas reflexões e confluências. Revista da ABENO, São Paulo, v. 7, n. 2, p.122-129, 2007.

GAMARRA JÚNIOR, J. S. Controle social na saúde: desafios e perspectivas. In: MACHADO, P. H. B.; LEANDRO, J. A.; MICHALISZYN, M. S. (Org.). Saúde coletiva: um campo em construção. Curitiba: Ibpex, 20o6. p. 149-176. 
MEDEIROS JÚNIOR, A.; RONCALLI, A. G. Políticas de saúde no Brasil. In: FERREIRA, M. A. F.;

RONCALLI, A. G.; LIMA, K. C. Saúde bucal coletiva: conhecer para atuar. Natal: EDUFRN, 2004. p. 249-

259.

VASCONCELOS, C. M. Uma análise entrelaçada sobre os paradoxos da mudança no SUS.

2005. Tese (Doutorado em Saúde Coletiva) -

Universidade Estadual de Campinas, Campinas, 2005 .

VASCONCELOS, C. M.; PASCHE, D. F. O Sistema Único de Saúde. In: CAMPOS, G. W. S. et al. (Org.). Tratado de saúde coletiva. São Paulo: Hucitec; Rio de Janeiro: Fiocruz, 20o6. p. 531-562.

VENTURA, D. F. L. Direito institucional sanitário. In: ARANHA, M. I. (Org.). Direito sanitário e saúde pública: coletânea de textos. Brasília, DF: Ministério da Saúde, 2003. p. 261-299. v.1.

VIANA, A. L. D.; LIMA, L. D.; OLIVEIRA, R. G. Descentralização e federalismo: a política de saúde em novo contexto: lições do caso brasileiro. Ciência e Saúde Coletiva, Rio de Janeiro, v. 7, n. 3, p. 493-507, 2002.

WHO. Constitution of the World Health Organization. Geneva, 1946. Disponível em: <http://whqlibdoc.who.int/hist/official_records/ constitution.pdf.>. Acesso em: 30 nov. 2009. 